\title{
In Vitro Demonstration of Synergy of Colistin with Various Antimicrobial Agents by Disc Diffusion and E Testing against Clinical Isolates of Carbapenem Resistant Acinetobacter
}

\author{
Sreekanth Reddy Basireddy ${ }^{1}$, Ahmed Sardar², Manisha Singh ${ }^{3}$, Vasanti Kabra ${ }^{4}$ \\ ${ }^{1}$ Assistant Professor, Department of Microbiology, ACSR Government Medical College, Nellore, Andhra Pradesh, India. \\ ${ }^{2}$ Assistant Professor, Department of Microbiology, SVS Medical College, Yenugonda, Telangana, India. ${ }^{3}$ Professor, \\ Department of Microbiology, SVS Medical College, Yenugonda, Telangana, India. ${ }^{4}$ Professor and HOD, Department of \\ Microbiology, SVS Medical College, Yenugonda, Telangana, India.
}

\section{ABSTRACT}

\section{BACKGROUND}

Acinetobacter is a notoriously drug resistant pathogen with many isolates being resistant to carbapenems. Colistin is often used in combination with other antibiotics as there is a chance of clinical failure when used as monotherapy. In vitro testing for synergy is extremely important for the clinician in order to choose the appropriate drug for treatment. The present study aims to evaluate the synergy of various antibiotics in combination with colistin.

\section{METHODS}

36 isolates of carbapenem resistant Acinetobacter were included in the study. Two groups of freshly prepared plates of plain Mueller Hinton agar (MH) and Mueller Hinton agar with $0.5 \mathrm{mcg} / \mathrm{ml}$ colistin were taken. $0.5 \mathrm{McF}$ arland suspension of the isolates was prepared, and swabbing was done on both the groups of $\mathrm{MH}$ agar plates. Disc diffusion testing was done by placing various antimicrobial agents like tigecycline, meropenem, cefoperazone/sulbactam rifampicin and ciprofloxacin on both the plates. Zones of inhibition were measured and differences in the zone sizes for these drugs with and without colistin combination were noted. All these isolates were further tested for synergy testing by using the E strips of these antibiotics on $\mathrm{MH}$ agar plates with and without colistin $(0.5 \mathrm{mcg} / \mathrm{ml})$ and comparing the minimum inhibitory concentrations (MIC) of these drugs. Synergy was considered when the difference is more than $5 \mathrm{~mm}$ by disc diffusion or when the MIC is decreased by 3 folds for that particular antibiotic when tested on $\mathrm{MH}$ agar plates with and without colistin.

\section{RESULTS}

Out of 36 isolates, highest synergy was observed for colistin in combination with rifampicin with 21 isolates (58.3\%) showing synergy. This is followed by combination with cefoperazone sulbactam (19 isolates, 52.7\%), meropenem (11 isolates, 30.5\%). Only 5 isolates (13.8\%) have shown colistin and tigecycline synergy. None of the isolates have shown synergy with ciprofloxacin. In 2 isolates synergy was observed for all the four drugs in combination with colistin. Good correlation was observed between disc diffusion and MIC testing with majority the isolates (81\%) showing more than $5 \mathrm{~mm}$ difference in the disc diffusion test showing synergies by MIC testing.

\section{CONCLUSIONS}

Disc diffusion testing by incorporating active drug in the medium may help in empirically choosing the appropriate combination for which additional tests can be chosen which are more accurate. This methodology is cost effective and easy.

\section{KEY WORDS}

Synergy, Colistin, Acinetobacter, Disc Diffusion

\author{
Corresponding Author: \\ Manisha Singh, \\ Professor, \\ Department of Microbiology, \\ SVS Medical College, \\ Yenugonda, Telangana, India. \\ E-mail: drsinghmanisha@gmail.com
}

DOI: $10.14260 / j e m d s / 2019 / 642$

Financial or Other Competing Interests: None.

How to Cite This Article:

Basireddy SR, Sardar A, Singh M, et al. In vitro demonstration of synergy of colistin with various antimicrobial agents by disc diffusion and E testing against clinical isolates of carbapenem resistant Acinetobacter. J. Evolution Med. Dent. Sci. 2019;8(39):2953-2957, $10.14260 /$ jemds/2019/642

Submission 06-08-2019,

Peer Review 14-09-2019,

Acceptance 20-09-2019,

Published 30-09-2019. 


\section{BACKGROUND}

Emergence of multidrug resistant pathogens has become a major healthcare issue. Acinetobacter baumannii is a notoriously drug resistant pathogen causing nosocomial infections like ventilator associated pneumonia, bacteraemia, skin and soft tissue infections and device associated infections in health care setups. Multi-drug resistance in these organisms has become a common occurrence and many of these Acinetobacter baumannii isolates are also showing increased resistance to carbapenems which are considered the best drugs for treatment. There are very few alternative drugs available for treatment of carbapenem resistant strains, among which colistin is an important option. Colistin is often used in combination with other antibiotics as there is a chance of clinical failure when it is used as monotherapy. ${ }^{1,2}$ In vitro testing for synergy is extremely important for the clinician in order to choose the appropriate drug for treatment. Many researchers have been working to standardize the In vitro methodologies of synergy testing. Though there are different in vitro synergy testing methodologies like time kill assays, checkerboard, E test methodologies, there is no consensus regarding which method should be used. Each and every method has its own advantages and disadvantages. Though time kill assays are suggested as the best methodologies for synergy detection, they are seldom used in routine microbiology laboratories because of the laborious and time-consuming procedures and they also need expertise. Among the entire methodologies, Etest based methodologies are considered simple and user friendly and requires less expertise but there has been no standardization of these methods and its correlation with time kill studies was highly variable in different studies. Above this, the synergy effects of various combinations of antibiotics against Acinetobacter baumannii is strain specific. So, each and every isolate needs to be tested separately by using various combinations of antibiotics to know which combination of antibiotics work better for that particular isolate. Testing for all the different combinations of antibiotics for each isolate is extremely difficult and also highly expensive because of which routine diagnostic laboratories cannot practice this for all the isolates. As disc diffusion methodology is the most widely practiced method for susceptibility testing for bacterial isolates even in resource poor settings, we have adopted the disc diffusionbased methodology in detecting the synergy of various antibiotics in combination with colistin against the carbapenem resistant Acinetobacter baumannii clinical isolates.

\section{METHODS}

This prospective study was done in SVS medical college, Mahbubnagar from July to December 2015. Only carbapenem resistant Acinetobacter baumannii isolates obtained during the study period were further processed for synergy testing. A total of 36 non repetitive isolates of carbapenem resistant Acinetobacter baumannii were obtained during the study period and were included in the study. Isolation and identification were done by routinely performed methods in our microbiology laboratory. Susceptibility testing was done by disc diffusion testing according to the CLSI Guidelines ${ }^{3}$. MICs of colistin were detected by E-strip methodology ( $\mathrm{Hi}$ Media). Pure powder of colistin was obtained from Sigma and was incorporated into Mueller Hinton agar plates at a fixed concentration of $0.5 \mu \mathrm{g} / \mathrm{ml}$.

For in vitro synergy testing, two groups of freshly prepared plates of plain Mueller Hinton agar (MH agar) and Mueller Hinton agar with $0.5 \mu \mathrm{g} / \mathrm{ml}$ colistin were taken. 0.5 McFarland suspension of the isolates was prepared, and swabbing was done on both the groups of $\mathrm{MH}$ agar plates. Disc diffusion testing was done by placing various antimicrobial agents like rifampicin, tigecycline, meropenem, cefoperazone/sulbactam and ciprofloxacin on both the plates. These antibiotics were chosen based on the data of the commonly used antibiotics in our hospital. Zones of inhibition were measured for all these antibiotics in both the plates and differences in the zone sizes for these drugs with and without colistin combination were noted. All these isolates were further tested for synergy testing by using the MIC strips of these antibiotics (HiMedia, India) on $\mathrm{MH}$ agar plates with and without colistin $(0.5 \mu \mathrm{g} / \mathrm{ml})$ and comparing the minimum inhibitory concentrations (MIC) of these drugs. Synergy was considered when the difference is more than $5 \mathrm{~mm}$ by disc diffusion or when the MIC is decreased by 3 folds for that particular antibiotic when tested on $\mathrm{MH}$ agar plates with and without colistin. ${ }^{4}$ E.coli ATCC 25922 and Pseudomonas aeruginosa ATCC 27853 were used as control strains. SPSS software was used to perform statistical analysis using a paired t-test. $\mathrm{p}<0.05$ was considered statistically significant.

\section{RESULTS}

Out of 36 isolates, highest synergy was observed for colistin in combination with rifampicin with 21 isolates (58.3\%) showing synergy by both disc diffusion and E test methods. This is followed by combination with cefoperazone/ sulbactam (19 isolates, 52.7\%), meropenem (11 isolates, $30.5 \%)$. only 5 isolates $(13.8 \%)$ have shown colistin and tigecycline synergy. None of the isolates have shown synergy with ciprofloxacin. In 2 isolates synergy was observed for all the four drugs in combination with colistin. Good correlation was observed between disc diffusion and MIC testing with majority the isolates (81\%) showing more than $5 \mathrm{~mm}$ difference in the disc diffusion test were showing synergies by MIC testing.

\begin{tabular}{|c|c|c|c|c|c|}
\hline 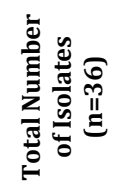 & ن & 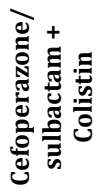 & 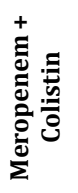 & 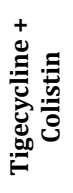 & 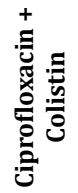 \\
\hline $\begin{array}{c}\text { Synergy } \\
\text { observed }\end{array}$ & 21 & 19 & 11 & 5 & 0 \\
\hline No synergy & 15 & 17 & 25 & 31 & 36 \\
\hline \multicolumn{6}{|c|}{$\begin{array}{c}\text { Table 1. Synergy of Various Antimicrobials } \\
\text { in Combination with Colistin }\end{array}$} \\
\hline
\end{tabular}




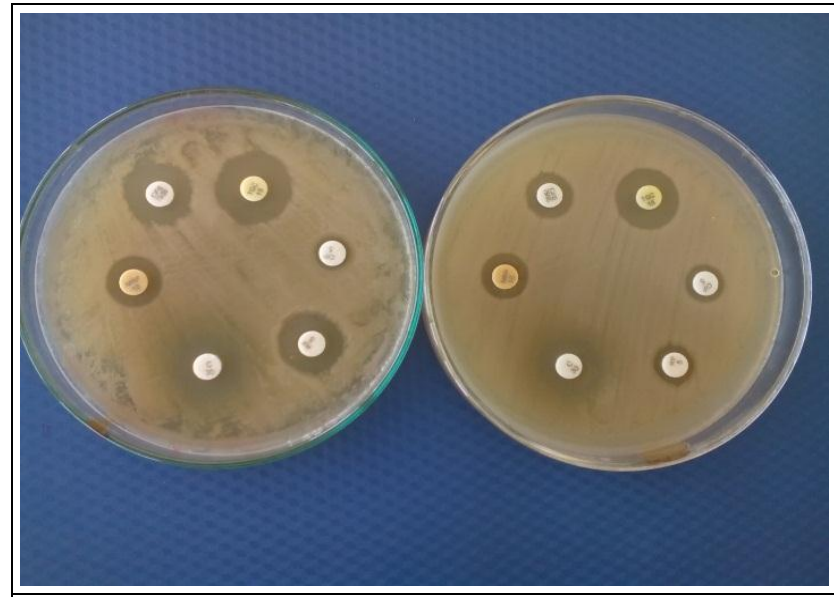

Figure 1. Disc Diffusion Testing on Muller Hinton Agar Plates with $(0.5 \mathrm{mcg} / \mathrm{ml})$ and without Colistin. Enhanced Zone of Inhibition was observed for some Antibiotics on Colistin Containing Agar (Left) Plate

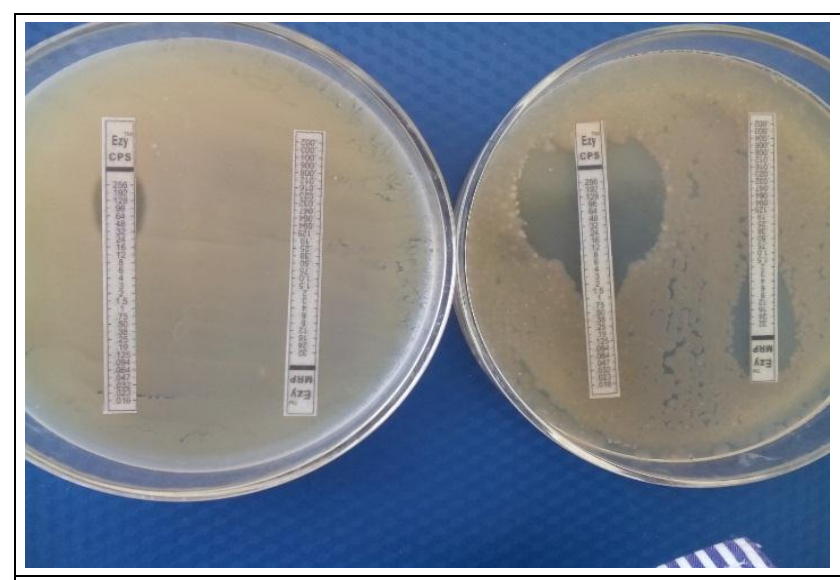

Figure 2. E Test/Agar Dilution Testing on Muller Hinton Agar Plates Plain and with Colistin $(0.5 \mathrm{mcg} / \mathrm{ml})$ Incorporated. Observe the Decrease in the MICs for Cefoperazone Sulbactam and Meropenem on Colistin Containing Agar Plate (Right Side Figure)

\section{DISCUSSION}

Acinetobacter is one of the most important nosocomial pathogens in hospitals which causes serious hospital acquired infections. Carbapenems have long been used as the drugs of choice for the treatment of these nosocomial infections. With the emergence of MDR Acinetobacter which is resistant to carbapenems too, the old and forgotten polymyxin group of drugs have gained importance once again with colistin being used extensively for the treatment of carbapenem resistant Acinetobacter. However, monotherapy with colistin is problematic as hetero resistance to colistin has been observed both in vitro and also during the therapy which raises concern about its monotherapy. Therefore, use of combination therapy has drawn attention of the clinicians for the treatment of MDR pathogens. Combination therapy helps in potentiating the effect of the other drugs and increases the eradication efficacy and also prevents the emergence of resistant population.

Synergy of colistin has been tested with various antibiotics like carbapenems, rifampicin, tigecycline, doxycycline, azithromycin etc by different researchers. ${ }^{4,5,6}$ In the present study colistin has shown maximum synergy with rifampicin (58.3\%). Many studies have evaluated colistin plus rifampicin synergy and they found it to be synergistic. In Tan et al ${ }^{7}$ study $56 \%$ of the drug resistant Acinetobacter baumannii isolates showed polymyxin $\mathrm{B}$ plus rifampicin synergy by time kill assays. In Wareham et $\mathrm{al}^{8}$ study all the strains showed reduced MICs with the polymyxin plus rifampicin combination by E test agar dilution methodology. Many in vivo studies have also demonstrated the effectiveness of colistin and rifampicin synergy in the treatment of drug resistant gram-negative bacteria.9,10,11 Next to rifampicin, cefoperazone sulbactam has shown good in vitro synergistic activity against Acinetobacter in our study. Though there are no CLSI guidelines regarding cefoperazone sulbactam, many clinicians use this drug in health care set up. The effectiveness of this drug against Acinetobacter is probably due to sulbactam which is known to have inherent activity against Acinetobacter. Other antibiotics like meropenem and tigecycline also showed synergistic effect to some extent in our study. Addition of colistin to meropenem might have potentiated the effect of meropenem by the action of the colistin on the outer membrane which allows meropenem to interfere with the cell wall synthesis. Carbapenems have long been considered as the most important and potent drugs against Acinetobacter species. But with the emergence of carbapenem resistance by various mechanisms, treatment of these infections become complicated. Colistin in combination with various carbapenems have been used for the treatment of nosocomial infections especially Acinetobacter species. Various researchers have also done invitro synergy testing of carbapenems in combination with colistin and have observed varying results. ${ }^{12,13}$ In a meta-analysis by Oren Zusman et al,12 synergy was observed for colistin and carbapenems in $77 \%$ of the Acinetobacter isolates by time kill assays and they have also observed antagonism in few isolates. In contrast in another study by David W Wareham et al marked synergy was not observed between polymyxin and imipenem when the synergy was done by E test methods against OXA 23 carbapenemase producing Acinetobacter isolates. In Lim et al $^{14}$ study polymyxin plus tigecycline has exhibited bactericidal activity in 9 out of 31 isolates (29\%). Whereas in Sopirala et $\mathrm{al}^{4}$ study no synergy was demonstrated with tigecycline plus colistin combination, but synergy/additivity was observed with imipenem plus colistin combination for all the isolates. Tetracycline group of drugs have been tested in combination with colistin by various researchers. ${ }^{7}$ Doxycycline and minocycline have shown good invitro synergy with colistin in their studies. The new derivative tigecycline belonging to glycylcyclines has shown different results in various studies when used in synergy. ${ }^{15,16}$

As colistin acts on the cell membrane, it disrupts the integrity of the membrane which makes the way for the hydrophilic antibiotics like rifampicin and vancomycin thus showing the synergistic activity with these drugs which are not having any action against Acinetobacter if used alone. Vancomycin, which is an antibiotic used against only grampositive organisms has shown good synergy with colistin against Acinetobacter in many studies ${ }^{17}$. Clinical experience regarding colistin treatment against the multidrug resistance Acinetobacter has not been always satisfactory. Poor outcomes have been noticed in many clinical studies with colistin which may be related to various factors like underlying illness severity, pharmacokinetics and 
pharmacodynamics of the drug, yet it is the only option available for the treatment in many cases. ${ }^{18,19,20}$

Multiple factors hinder the synergy testing including the tested strains, mechanisms of resistance, type of the methodology used and also the quality of the working environment. Each and every method of synergy testing has its own advantages and disadvantages. As of now, none of the synergistic methods have been standardized and there is an urgent need of standard and easy method for rapid identification of synergy between various antimicrobials which guides the clinicians in real time so that appropriate treatment can be given, which further decreases the mortality and morbidity and the huge economic burden associated with these resistant infections in the hospitals.

\section{CONCLUSIONS}

Disc diffusion testing by incorporating active drug in the medium may help in empirically choosing the appropriate combination for which additional tests like time kill studies, checkerboard assays or E-test based methodologies can be chosen which are more accurate. This methodology is quick, cost effective and helps in decreasing the laborious work. Timely identification of the multidrug resistant organisms and determining the appropriate treatment regimens by available synergy testing in association with proper infection control practices will not only decrease the morbidity and mortality associated with serious nosocomial infections but also help in preventing the spread of these infections which further adds to economic burden on the health care system.

\section{Disclosure}

This work was presented as poster in $26^{\text {th }}$ ECCMID conference in Amsterdam, Netherlands (2016)

\section{REFERENCES}

[1] Hawley JS, Murray CK, Jorgensen JH. Colistin heteroresistance in Acinetobacter and its association with previous colistin therapy. Antimicrob Agents Chemother 2008;52(1):351-2.

[2] Rodriguez CH, Bombicino K, Granados G, et al. Selection of colistin-resistant Acinetobacter baumannii isolates in postneurosurgical meningitis in an intensive care unit with high presence of heteroresistance to colistin. Diagn Microbiol Infect Dis 2009;65(2):188-91.

[3] Clinical and Laboratory Standards Institute. Performance standards for antimicrobial susceptibility testing; 20th informational supplement. CLSI document M100-S20. Clinical and Laboratory Standards Institute, Wayne, PA. 2010.

[4] Sopirala MM, Mangino JE, Gebreyes WA, et al. Synergy testing by Etest, microdilution checkerboard and timekill methods for pan-drug-resistant Acinetobacter baumannii. Antimicrob Agents Chemother 2010;54(11):4678-83.
[5] Timurkaynak F, Can F, Azap OK, et al. In vitro activities of non-traditional antimicrobials alone or in combination against multidrug-resistant strains of Pseudomonas aeruginosa and Acinetobacter baumannii isolated from intensive care units. Int J Antimicrob Agents 2006;27(3):224-8.

[6] Miyasaki Y, Morgan MA, Chan RC, et al. In vitro activity of antibiotic combinations against multidrug resistant strains of Acinetobacter baumannii and the effects of their antibiotic resistance determinants. FEMS Microbiology Letters 2012;328(1):26-31.

[7] Tan TY, Lim TP, Lee WH, et al. In vitro antibiotic synergy in extensively drug-resistant Acinetobacter baumannii: the effect of testing by time-kill, checkerboard and Etest methods. Antimicrob Agents Chemother 2011;55(1):436-8.

[8] Wareham DW, Bean DC. In-vitro activity of polymyxin B in combination with imipenem, rifampicin and azithromycin versus multidrug resistant strains of Acinetobacter baumannii producing OXA-23 carbapenemases. Ann Clin Microbiol Antimicrob 2006;5:10.

[9] Aydemir H, Akduman D, Piskin N, et al. Colistin vs. the combination of colistin and rifampicin for the treatment of carbapenem-resistant Acinetobacter baumannii ventilator-associated pneumonia. Epidemiol Infect 2013;141(6):1214-22.

[10] Tsioutis C, Kritsotakis EI, Maraki S, et al. Infections by pandrug-resistant gram-negative bacteria: clinical profile, therapeutic management and outcome in a series of 21 patients. Eur J Clin Microbiol Infect Dis 2010;29(3):301-5.

[11] Falagas ME, Rafailidis PI, Matthaiou DK, et al. Pandrugresistant Klebsiella pneumoniae, Pseudomonas aeruginosa and Acinetobacter baumannii infections: characteristics and outcome in a series of 28 patients. Int J Antimicrob Agents 2008;32(5):450-4.

[12] Zusman O, Avni T, Leibovici L, et al. Systematic review and meta-analysis of in vitro synergy of polymyxins and carbapenems. Antimicrob Agents Chemother 2013;57(10):5104-11.

[13] Wareham DW, Bean DC. In-vitro activity of polymyxin B in combination with imipenem, rifampicin and azithromycin versus multidrug resistant strains of Acinetobacter baumannii producing OXA-23 carbapenemases. Annals of Clinical Microbiology and Antimicrobials 2006;5:10.

[14] Lim TP, Tan TY, Lee W, et al. In-vitro activity of polymyxin B, rifampicin, tigecycline alone and in combination against carbapenem-resistant Acinetobacter baumannii in Singapore. PLoS One 2011;6(4): e18485.

[15] Arroyo LA, Mateos I, González V, et al. In vitro activities of tigecycline, minocycline and colistin-tigecycline combination against multi- and pandrug-resistant clinical isolates of Acinetobacter baumannii group. Antimicrobial Agents and Chemotherapy 2009;53(3):1295-6.

[16] Dizbay M, Tozlu DK, Cirak MY, et al. In vitro synergistic activity of tigecycline and colistin against XDRAcinetobacter baumannii. The Journal of Antibiotics (Tokyo) 2010;63(2):51-3. 
[17] Gordon NC, Png K, Wareham DW. Potent synergy and sustained bactericidal activity of a vancomycin-colistin combination versus multidrug-resistant strains of Acinetobacter baumannii. Antimicrobial Agents and Chemotherapy 2010:54(12):5316-22.

[18] Kroeger LA, Hovde LB, Mitropoulos IF, et al. Colistin methanesulfonate against multidrug-resistant Acinetobacter baumannii in an in vitro pharmacodynamic model. Antimicrob Agents Chemother 2007;51(9):3431-3.
[19] Livermore DM, Hill RL, Thomson H, et al. Antimicrobial treatment and clinical outcome for infections with carbapenem- and multiply-resistant Acinetobacter baumannii around London. Int J Antimicrob Agents 2010;35(1):19-24.

[20] Wareham DW, Bean DC, Khanna P, et al. Bloodstream infection due to Acinetobacter spp: epidemiology, risk factors and impact of multi-drug resistance. Eur J Clin Microbiol Infect Dis 2008;27(7):607-12. 